Document downloaded from:

http://hdl.handle.net/10251/168745

This paper must be cited as:

Giret Boggino, AS.; Julian Inglada, VJ.; Botti, V. (2019). An Intelligent Platform for supporting optimized collaborative urban logistics. Springer. 3-14. https://doi.org/10.1007/978-3-03027477-1_1

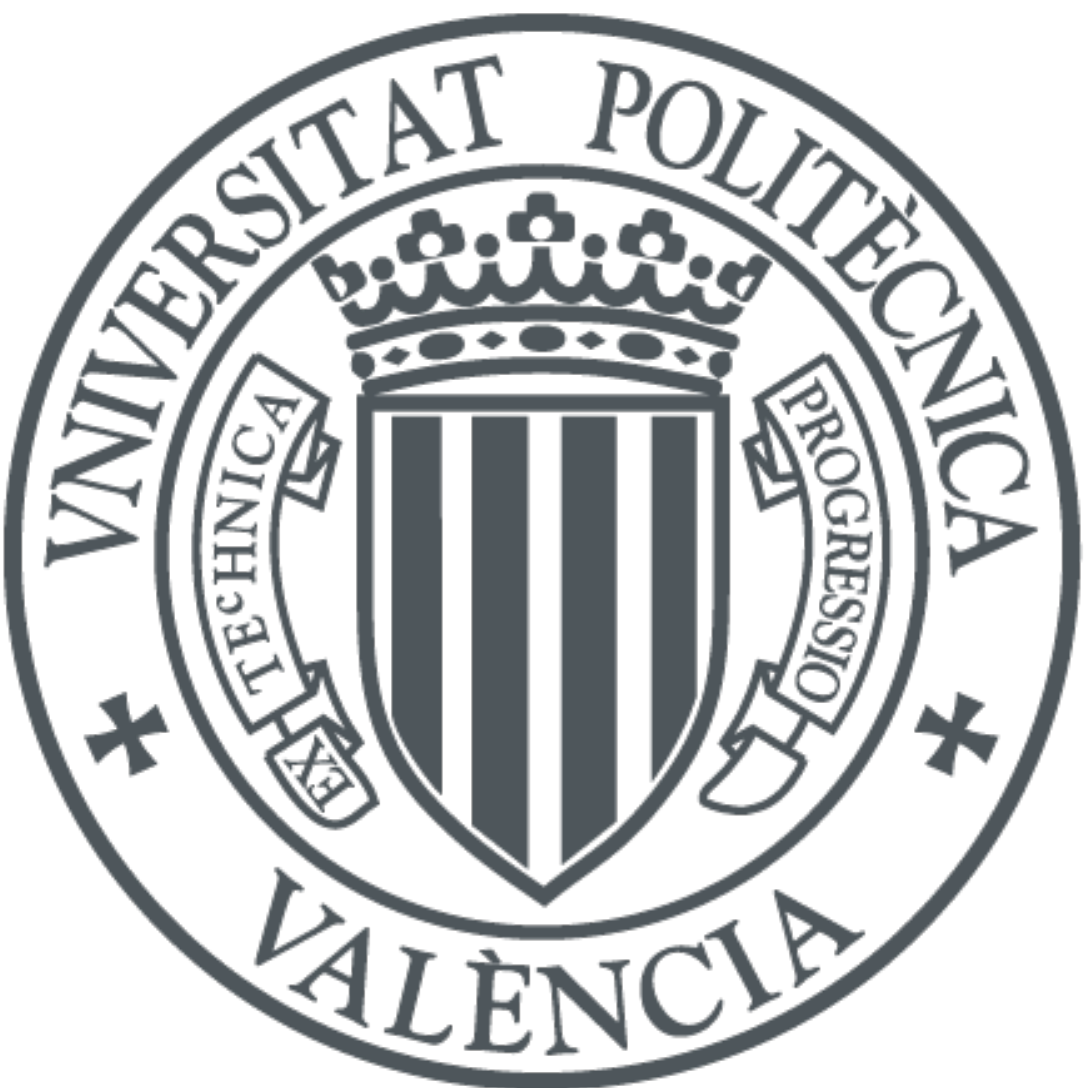

The final publication is available at

https://doi.org/10.1007/978-3-030-27477-1_1

Copyright Springer

Additional Information 


\title{
An Intelligent Platform for supporting optimized collaborative urban logistics
}

\author{
Adriana Giret*, Vicente Julián, and Vicente Botti \\ Universitat Politècnica de València, Valencia, Spain.
}

\begin{abstract}
Optimized urban logistics is an important issue for rapidly growing cities worldwide. Many criteria can be optimized in order to improve the performance of urban logistics. Economic and time dependent criteria are central but not the only ones, lately, sustainable criteria are becoming key and urgent due to new regulations and environmental concern of governments and the society. In this work we review the state of the art of intelligent developments and techniques that might aid to build smart and optimized urban logistic applications. Moreover, we propose a prototype platform conceived as a supporting and facilitating layer for the growing business of last mile delivery (LMD) companies that operate in cities in an isolated way. Our vision is to provide a cooperative intelligent platform that provides coordination and collaboration services for the LMD companies of urban areas.
\end{abstract}

Keywords: Urban Logistics, Green Last Mile Delivery, Cooperative Logistics

\section{Introduction}

European cities (as most of worldwide cities) are forced to tackle a wide range of urban traffic problems: first of all the big challenge of reducing traffic congestions, $\mathrm{CO} 2$, pollutant emissions, and energy consumption. According to the European Environment Agency, cities emit 69\% of Europe's CO2 and urban transport accounts for $70 \%$ of the pollutants and $40 \%$ of the greenhouse gas emissions from European road transport (European Environment Agency). On the other hand, cities have to guarantee to citizens not only the overall accessibility to the different city and transport services, but also an efficient urban logistic with respect to the economic and environmental factors. According to this, the Transport Policy White Paper ${ }^{1}$ set up the $\mathrm{CO} 2$ free urban logistics as one of the 10 objectives to reach by 2030. The EU guidelines for "Developing and Implementing a Sustainable Urban Mobility Plan", aims to provide realistic and simple guidelines for city stakeholders and technicians, for developing a Sustainable Urban Logistics Plan focused on the optimization of urban logistics

\footnotetext{
* Corresponding Author, agiret@dsic.upv.es

${ }^{1}$ https://www.eesc.europa.eu/sites/default/files/resources/docs/pp-white-papertransport-may16-en.pdf
} 
processes in order to reduce the related energy consumption and environmental impacts yielding its economic sustainability.

Citizens' demands are increasing and accordingly, there is a considerable load on developing efficient distributive logistics. One sector that is growing very fast, mainly due to on-line retail, is Last Mile Delivery (LMD). LMD is a term used in supply chain management and transportation planning to describe the movement of people and goods from a transportation hub to a final destination in the home. In general the delivery area is defined as the city/urban area. In 2018, the Global Last Mile Delivery market size was 30200 million US Dollars and it is expected to reach 55200 million US Dollars by the end of 2025, with a CAGR of $9.0 \%$ during 2019-2025 [13]. In the business to customer (B2C) e-commerce context, final delivery is one of the most complicated, expensive and inefficient segments along the whole logistics fulfillment chain [16].

Recent studies and commercial data demonstrate that technology focused start-up companies have painted a target on LMD. From freight forwarders to warehouse analytics platforms, delivery and logistics providers, over 350 supply chain management and logistics ventures are listed on AngelList (https://angel.co), the online network of start-up ventures, investors and job seekers. The increase in supply chain and logistics start-up ventures coincides with changes in the supply chain due to omnichannel and e-commerce fulfillment needs. No longer are online retailers limited to dealing with the same carriers for package delivery. Parcel delivery can now be handled by many other innovative vendors servicing the online shopping industry. Is this the future of retail? Quite possibly. This tremendous growth comes with associated coordination problems that, to the best of our knowledge, have yet to be solved. In today's developments of solutions and companies for LMD there is a lack of a common support that can aid in facilitating the interaction/coordination and cooperation among these, most of the time, isolated LMD companies/actors. Having that kind of facilitating/cooperation framework can improve and optimize the LMD overall performance into a given city/urban zone. Think for example on one of the main goals of the Smart Cities paradigm: "to ensure the sustainable development with the aid of technologies" [10]. Now picture a scenario in which there are a large number of individual deliverers (working for different LMD companies) that navigate the city in order to deliver packages and when doing it, most of the times, the same area/delivery path is navigated many times by different deliverers in order to reach their delivery destination. Is it possible to optimize this scenario taking into account the principles of sustainable development? We believe the answer to this question is Yes. Our vision relays on a coordination/facilitating intelligent platform layer that can aid to increase the delivery route packagecapacity-cost-rate (it is important to point out that this rate can be parameterized including security/capacity thresholds, quality of service specification, etc.). The approach will foster and facilitate sub-contracting delivery services to those deliverers (from LMD companies to LMD companies, i.e. a B2B approach) with optimized package-capacity-cost-rate that will navigate the destination area of the package instead of contracting a new deliverer that will execute the same 
route. In this way the number of dedicated movements will be decreased with the added effect of reducing $\mathrm{CO} 2$ emissions and traffic congestion.

\section{Last Mile Delivery and Sustainable Urban Logistic}

Cities are places of consumption, production, and distribution of material goods. Urban logistics [5] includes all activities ensuring that the material demands of these activities are satisfied. It includes all goods movements generated by the economic needs of local businesses, that is, all deliveries and collection of supplies, materials, parts, consumables, mail and refuse that businesses require to operate [8]. As a city hosts a great number of different economic sectors, it is provisioned by hundreds of different supply chains, making urban logistics very complex and diverse. Over the past two decades delivering goods into cities has become a challenge with cities getting overly congested and traffic jams resulting in expensive logistics bottlenecks. Studies show that the cost of congestion now in terms of time wasted in traffic and fuel consumption is very high, almost $200 \%$ more than what it was in the 1980s. Pollution, lack of parking bays, and warehousing costs are all restraints that are contributing to the economic cost of urban logistics.

The concept of Sustainable Urban Logistic (or Sustainable Last Mile Logistic) is closely related with that of Sustainable Supply Chains. A Sustainable Supply Chain is one that performs well on both traditional measures of profit and loss as well as on an expanded conceptualization of performance that includes social and natural dimensions [11]. In the specialized literature a large number of works related with measuring the economic performance of logistics solutions can be found. Nevertheless, less works that mix all the aspects of sustainable logistics were reported. Among the few, but interesting works the list of 21 indicators proposed by Morana in [9] is one of the most influential and useful studies. The Morana list is in turn built from two other works [7] and [6].

Although, passenger travel have received most of the attention $[4,1]$, other contributions to new research and technology are found for the modeling of delivery of parcels (see [10] for a state of the art review on requirements and features of transport, mobility, and logistics in Smart Cities).

In [12] the author proposes a Multi Agent System simulation model capable of optimizing the distribution phase of small and medium packaged parcels in supply chains management and investigates if improvements in efficiency of time, cost and energy can be achieved. In his proposal the author uses public transport and e-cargo bikes in a combined way for the delivery of parcels. In [15] the authors propose a crowdserving approach for LMD for birck-and-mortar retailers, in which the customers that visit the retail stores are asked to deliver orders, by means of an phone App, to other customers that live near their neighborhood. Another interesting approach is described in [15], where a Multi Agent System framework is proposed in order to support citizens that are willing to deliver parcels using their own routes to the work, to the university etc. when the delivery address is on their way. The proposed approach provides an intermodal 
planning engine for transportation network analysis in order to prioritize those citizens that move by means of eco-efficient vehicles and public transportation systems.

Another related work is [3], that discusses the benefits of using new alternative delivery options as opposed to conventional delivery modes (for instance cars and vans). The author describes the success, in pilot tests, of Micro-Carrier Urban Vehicle (MCUV). A commercial application that follows this approach is the solution from DPD parcel delivery brand (https://www.dpd.com/) that provides a set of services and options for smart urban delivery: an app that accurately informs the users about their shipments delivery times and allows changing or redirecting them if necessary; an alternative zero emission drive system with electric or hybrid vehicles in Stuttgart, and transport bikes in Hamburg. Another example is Green Link ( $h t t p: / / g r e e n-l i n k . c o . u k$ ), in which an emission-free delivery solution that uses electric vehicles, load-carrying tricycles, bicycles and load-carrying trailers for last mile delivery of parcels from their centres to the final destination is provided. The courier companies deliver the parcels to Green Link centers (similar to UDC) and from this point Green Link completes the distribution with their own deliverers and emission-free vehicles to the final customer. Green Link operates in York, Luton and Darlington. Other examples of self-service parcel station are DHL PackStation, LaPoste Pickup Station etc.

In order to understand the huge problem that a large market of LMD companies can entail to big cities let's look at China's LMD market [16]. The booming e-commerce industry in China is witnessing high growth rates, it is foreseeing that by 2023 the market size will be over 60 billion US Dollars. This requires the parallel development of the last mile delivery services driving the growth of the market. With growing internet and smartphone penetration, e-commerce is becoming fundamental, which is the primary driver for the development of last mile delivery market. According to China's Ministry of Commerce, the online retail sales in the country increased by $32 \%$ in 2017 . The express delivery market of the country is the largest in the world and accounts for almost $40 \%$ of the total delivery volume across the globe. The sector also created many jobs; the number of employees increased by $130 \%$ between January 2014 and November 2017. These trends in China have lead to a very large list of express delivery companies that operate in all the big cities. Some of the major players include: SF Express (Group) Co. Ltd, Cainiao Logistics (with over two million delivery drivers in the network), ZTO Express (Cayman) Inc., Shanghai Yuantong Express Co. Ltd/YTO Express, STO Express Co.Ltd, Best Inc., China Post Express Logistics Co. Ltd/Express Mail Service (EMS), Deppon Logistics Co. Ltd, Alibaba (that holds a controlling interest in logistics operator Cainiao) and JD.com ${ }^{2}$, among others. Chinese couriers deliver an astonishing number of parcels every day, over 100 million per day, and three times what the USA delivers. Next-day and same-day delivery are standard. Some experts already feel

\footnotetext{
${ }^{2}$ Alibaba is opening a trade hub in Belgium in partnership with the government, and JD.com is opening a hub in France. Both companies are targeting European merchants that want to sell into the Chinese market.
} 
that the e-commerce logistics solutions available in China are world-leading, and we believe they may well be right.

\section{The Intelligent Platform for collaborative urban logistic}

In this section the platform for collaborative urban logistic is described. The proposed platform relies on intelligent techniques in order to support the collaborative coordination among LMD companies that simultaneously work in a city (a B2B coordination). The features that drive the collaboration among the companies may be multiple, i.e. economic, environmental, temporal, customer satisfaction, etc. The proposed approach allows using different and/or combined optimization criteria. In this paper we focus on the package-capacity of delivery routes in order to describe the gainings when using collaborative urban logistic approaches for optimizing the criteria.

\subsection{Definitions}

A LMD company has a delivery staff of delivery drivers that might be static or dynamic (new drivers might enter or exit the team). Each delivery driver is responsible of a set of packages to deliver in a single delivery route. The delivery route is defined by the set of packages assigned to the given deliverer. The packages from this set may (normally) have different destination addresses. The driver will stop along the route in order to drop the packages to the receiver customers. In order to optimize the process the set of packages assigned to the driver should build a delivery route with an optimized package-capacity-cost rate. Moreover, during the delivery execution new packages can enter the set of packages to deliver which implies the delivery route might change dynamically.

The big picture appears when all the LMD companies are executing in the same urban area. The number of delivery routes that run in this scenario is large and, in many cases, very similar delivery routes are executed many times. A collaborative urban logistic takes advantage of similar routes in order to minimize those with a bad package-capacity-cost rate.

The market of LMD companies that simultaneously work in an urban area is: $L M D-$ Market $=\left\{C_{1}, \ldots, C_{N}\right\}$, where $C_{i}$ is a company, $N>1$ is the number of companies that work in the city.

A LMD company $C_{i}$ is defined by its set of delivery drivers: $C_{i}=\left\{d_{C_{i} 1}, \ldots, d_{C_{i} j}\right\}$, where $d_{C_{i} i}$ is a delivery driver, $j>1$ is the number of drivers in the delivery staff of the LMD company.

The set of packages assigned to the deliverer $x$ from LMD company $C_{i}$ is defined as: $P S_{d_{C_{i} x}}=\left\{p_{1}, \ldots, p_{z}\right\}$, where $p_{i}$ is a package, $z>=1$ is the number of packages in the set assigned to driver $d_{C_{i} x}$.

The delivery route for a driver $x$ from LMD company $C_{i}$ is defined as: 
$d_{C_{i} x}$.route $=\left\{s_{1}, \ldots, s_{k}\right\}$, where $s_{i}$ is a destination address of a package from the set $P S_{d_{C_{i} x}}$. In other words a stop in the delivery route to drop a package. $k=z$, the number of stops is equal to the number of packages in the set.

The package-capacity of delivery route $d_{C_{i} x}$.route is calculated as:

capacity $\left(d_{C_{i} x}\right.$.route $)=\left(\right.$ Volume $\left.\left(P S_{d_{C_{i} x}}\right) * 100\right) / d_{C_{i} x}$.Capacity,

where Volume $\left(P S_{d_{C_{i} x}}\right.$ is calculated adding the volume of every package in the set, and $d_{C_{i} x}$. Capacity is the transportation capacity of the vehicle that is used by driver $d_{C_{i} x}$.

Finally the package-capacity-cost rate of delivery route $d_{C_{i} x}$.route is calculated as:

CapacityCostRate $\left(d_{C_{i} x}\right.$. route $)=\operatorname{capacity}\left(d_{C_{i} x}\right.$. route $) / \operatorname{Cost}\left(d_{C_{i} x}\right.$.route $)$,

where $\operatorname{Cost}\left(d_{C_{i} x}\right.$.route $)$ is calculated from the delivery route length and number of stops. It is important to point out that the calculation function will depend on the criterion or multiple criteria used to measure the cost. The typical economic criterion may be used, solely or combined with others criteria such as environmental, temporal, customer satisfaction, etc

The idea behind our approach is to use the set $L M D-$ Market of different companies with their large set of drivers that executes different delivery routes in order to search and optimize the package-capacity-cost rate of the delivery routes. In the following sections the intelligent platform that implements this idea is described.

\subsection{Conceptual Architecture}

In order to support the collaboration among different LMD companies we propose a layered architecture that incorporates intelligent modules for calculating and optimizing the collaborative routes.

Figure 1 shows the proposed architecture. The $L M D-$ Market is the ecosystem of companies $C_{i}$ that can use the lower intelligent supporting modules in order to complete their delivery services. The platform can run in two combined modes: push and pull. The push mode is when the LMD companies request collaboration to/from others LMD companies. The pull mode is when the platform intelligent modules recommend/seek collaboration from LMD companies. Both execution modes are always motivated by the need for optimizing the CapacityCostRate.

In the push mode, the LMD companies may submit two types of calls:

1. A CallForDelivery is issued by a company when it has a package that worsen the CapacityCostRate of its delivery staff of drivers. The best option for the company is to offer it to other companies because there could be one company that has a delivery route that goes to the address area of the given package. The CallForDelivery describes the package address, volume, delivery time to customer and the price offered for the delivery service. 


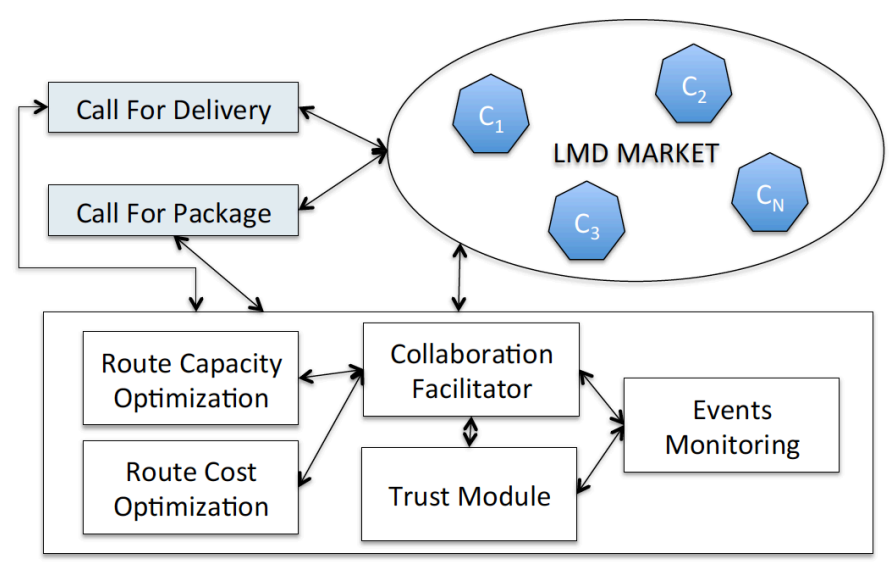

Fig. 1. Conceptual Architecture of the Intelligent Platform for collaborative Urban Logistic

2. A CallForPackage, in order to offer a slot (space) into a delivery route set of packages. This call is triggered by a LMD company when it realices that a given delivery route from its delivery staff of drivers has a bad CapacityCostRate. The CallForPackage describes the volume of the slot available, the address area that the given delivery route will visit, the cost requested for the offered delivery service. It is important to point out that the requested cost will normally be low because the LMD company offering the service is trying to optimize the route CapacityCostRate. Other important data that complete the CallFor Package is the deadline of the call, and the delivery time window for the customer.

Both types of calls are managed by the intelligent platform. The specific module that supports them is the CollaborationFacilitator. This module seeks among the other LMD companies in order to find those delivery routes that can be appropriate to the call. The CollaborationFacilitator uses the modules RouteCapacityOptimization and RouteCostOptimization in order to find the delivery routes that could enhance their CapacityCostRate by adding a new package to their set. When a company is interested in the call a negotiation process is started in which the two companies can negotiate some parameters of the package delivery. In general the most important parameter in the negotiation will be the service cost, nevertheless other parameters such as delivery time to customer could be also subject to negotiation.

In the pull mode, the intelligent platform initiates the calls/offers to the LMD companies when the platform detects delivery routes with bad CapacityCostRate. The EventsMonitoring module identifies the routes that requires optimization and requests collaboration from RouteCapacityOptimization and RouteCost Optimization in order to find good candidates to enhance the routes. These 
modules use intelligent metaheuristic search techniques [2] in order to find the good candidates. The CollaborationFacilitator is responsible to submit the recommendation to the affected LMD companies. In order to compose the offers the CollaborationFacilitator appends trust information facilitated by the TrustModule. The TrustModule uses a multi-agent trust and reputation model [14] that keeps record of the performance of the delivery drivers and LMD companies in the LMD - Market. The affected LMD companies uses the trust information in order to better decide to participate in the collaborative urban logistic. When the LMD companies agrees to negotiate the exchange of packages, a negotiation process starts. Similar to the push mode the delivery parameters can be negotiated among the parties. The CollaborationFacilitator manages the negotiation process.

\section{Experimentation}

A prototype implementation of the proposed platform was executed in order to measure its performance. It is important to point out that the intelligent algorithms for the modules RouteCapacityOptimization, RouteCostOptimization and TrustModule are the proposed in [2] and [14], nevertheless other algorithms are possible. The used negotiation protocol was the two rounds Face to Face protocol in which the parties negotiate the cost of the delivery service, all the same, others negotiation protocols and criteria can also be used.

The experimentation Case Study is defined as follows:

$L M D-$ Market $=\left\{C_{1}, \ldots, C_{10}\right\}, N=10$, i.e. 10 LMD companies,

$C_{i}=\left\{d_{1}, \ldots, d_{60}\right\}$, where $1<=i<=10$, i.e. each company has 60 delivery drivers,

$P S_{d_{C_{i} x}}=\left\{p_{1}, \ldots, p_{20}\right\}$, where $1<=i<=10$ and $1<=x<=60$, i.e. each driver has a package set of 20 packages. It is important to point out that the experimentation executed two packages sets for each driver. In this way a simplified simulation of two delivery rounds were executed, trying to mimic morning and afternoon shift.

The specification of: vehicle volume capacity for delivery packages transportation; packages specification about volume and delivery address, and; the delivery cost of every package, where generated randomly. It is important to point out that the vehicle volume capacity for every vehicle was generated taking into account the maximum volume, $\max \left(\operatorname{Volume}\left(P S_{d_{C_{i} x}}\right)\right.$, of all the generated package sets. In this way the vehicle volume capacity is a random number between $\max \left(\operatorname{Volume}\left(P S_{d_{C_{i} x}}\right)\right.$ and $2 * \max \left(\operatorname{Volume}\left(P S_{d_{C_{i x}}}\right)\right.$.

A route generation module was used in order to generate the shortest route with 20 or less stops (since it is possible to have two or more packages in the same package set with the same delivery address). The module builds the route using the Dijkstra's shortest path algorithm.

For the execution rounds $20 \%$ of push CallForDelivery and CallFor Package were simulated. The other cooperation intentions were activated by pull calls from the intelligent platform. 


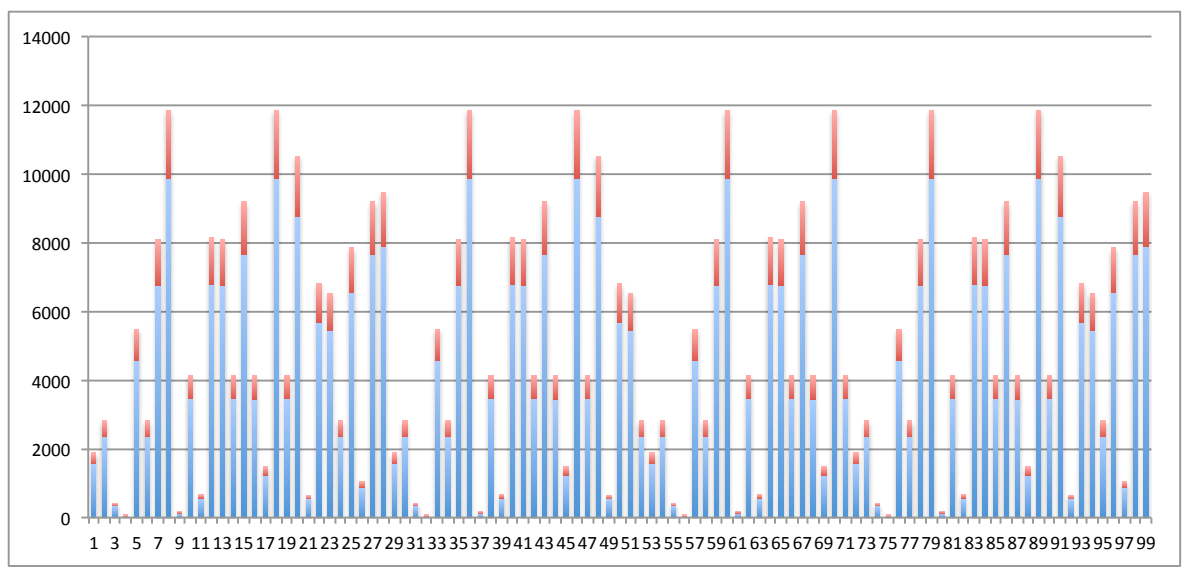

Fig. 2. Number of Interchanged Packages for the 100 execution rounds

100 execution rounds were ran in order to measure the behaviour of the system. Figure 2 depicts the number of packages that were interchanged among the drivers of the different LMD companies. The red section of the bars in the figure are from the push calls initiated by the LMD companies.

Figure 3 and 4 detail the delivery data of the 24000 packages, simulated in 100 rounds. The first column is the cost of delivering 24000 packages without interchange. The second column is the number of packages that are interchanged among drivers of different LMD companies. The third column is the cost of delivering the interchanged packages. The fourth column is the gaining cost of executing the collaborative delivery. The fifth column is the average gaining. The data confirm an average $11,5 \%$ reduction of delivery economic cost of the LMD market.

\section{Conclusion and Future Works}

In this paper an intelligent platform for optimized collaborative urban logistic was proposed. It follows a layered architecture approach in which different modules try to optimize the delivery routes from different LMD companies. The platform support a LMD market of companies that can operate in two modes or strategies: push, pull. The push mode is when the LMD companies request collaboration to/from others LMD companies. The pull mode is when the platform intelligent modules recommend/seek collaboration from LMD companies. In this paper we used the package-capacity-cost of delivery routes in order to describe the gainings when using collaborative urban logistic approaches for optimizing delivery criteria such as: economic, environmental, temporal, customer satisfaction, etc. In order to test the performance of the proposed approach a prototype implementation was executed with synthetic data from a set of 10 LMD companies that work in a city. The results show that the package-capacity-cost of 


\begin{tabular}{|c|c|c|c|c|}
\hline Delivery Cost (€) & № Interchange & Interchange Cost (€) & Cost Gaining (€) & Average Gaining (\%) \\
\hline 19200 & 1566 & 156,6 & 1096,2 & 4,35 \\
21600 & 2345 & 234,5 & 1876 & 8,38 \\
7200 & 345 & 34,5 & 172,5 & 0,72 \\
12000 & 23 & 2,3 & 6,9 & 0,08 \\
4800 & 4567 & 456,7 & 1370,1 & 16,65 \\
14400 & 2345 & 234,5 & 1641,5 & 8,14 \\
21600 & 6754 & 675,4 & 2026,2 & 24,12 \\
14400 & 9876 & 987,6 & 5925,6 & 35,27 \\
16800 & 125 & 12,5 & 12,5 & 0,00 \\
2400 & 3456 & 345,6 & 345,6 & 12,60 \\
2400 & 564 & 56,4 & 0 & 1,57 \\
14400 & 6785 & 678,5 & 2714 & 0,00 \\
12000 & 6754 & 675,4 & 3377 & 25,01 \\
2400 & 3456 & 345,6 & 1728 & 12,00 \\
4800 & 7654 & 765,4 & 5357,8 & 26,58 \\
2400 & 3445 & 344,5 & 1378 & 11,96 \\
9600 & 1234 & 123,4 & 987,2 & 4,11 \\
9600 & 9876 & 987,6 & 0 & 36,01 \\
7200 & 3456 & 345,6 & 0 & 12,60 \\
9600 & 8766 & 876,6 & 6136,2 & 32,47 \\
16800 & 543 & 54,3 & 434,4 & 1,81 \\
12000 & 5667 & 566,7 & 566,7 & 18,89 \\
19200 & 5439 & 543,9 & 1631,7 & 19,43 \\
12000 & 2345 & 234,5 & 0 & 8,38 \\
2400 & 6545 & 654,5 & 3927 & 23,38 \\
4800 & 876 & 87,6 & 613,2 & 2,43 \\
14400 & 7654 & 765,4 & 6123,2 & 0,00 \\
14400 & 7887 & 788,7 & 5520,9 & 21,91 \\
7200 & 1566 & 156,6 & 626,4 & 5,22 \\
14400 & 2345 & 234,5 & 703,5 & 0,00 \\
7200 & 345 & 34,5 & 241,5 & 0,00 \\
21600 & 23 & 2,3 & 0 & 0,08 \\
21600 & 4567 & 456,7 & 1826,8 & 15,86 \\
7200 & 2345 & 234,5 & 469 & 7,33 \\
12000 & 6754 & 675,4 & 4727,8 & 18,76 \\
2400 & 9876 & 987,6 & 0 & 0,00 \\
19200 & 125 & 12,5 & 62,5 & 0,46 \\
4800 & 3456 & 345,6 & 2073,6 & 11,52 \\
7200 & 564 & 56,4 & 169,2 & 1,96 \\
16800 & 6785 & 678,5 & 1357 & 24,23 \\
2400 & 6754 & 675,4 & 2701,6 & 21,11 \\
14400 & 3456 & 345,6 & 2073,6 & 0,00 \\
19200 & 7654 & 765,4 & 3061,6 & 26,58 \\
21600 & 3445 & 344,5 & 344,5 & 12,76 \\
7200 & 1234 & 123,4 & 493,6 & 4,11 \\
19200 & 9876 & 987,6 & 7900,8 & 35,27 \\
16800 & 3456 & 345,6 & 2073,6 & 9,60 \\
2400 & 8766 & 876,6 & 1753,2 & 27,39 \\
\hline & & & & \\
\hline
\end{tabular}

Fig. 3. Experiment Data. Part 1

delivery routes is a good measure in order to optimize the routes. An average $11,5 \%$ reduction of delivery economic cost of the LMD market was achieved by the implemented algorithms. As future works a dynamic module for on the fly support of failure events is foreseen in order to cope with undelivered packages. An enhanced negotiation protocol module that provides a full library of different negotiation strategies will be added to the platform. A library of optimization algorithms supported by big data searching techniques will also be included. 


\begin{tabular}{|c|c|c|c|c|}
\hline Delivery Cost (€) & № Interchange & Interchange Cost (€) & Cost Gaining (€) & Average Gaining (\%) \\
\hline 14400 & 543 & 54,3 & 162,9 & 0,00 \\
\hline 2400 & 5667 & 566,7 & 0 & 20,99 \\
\hline 19200 & 5439 & 543,9 & 3263,4 & 0,00 \\
\hline 7200 & 2345 & 234,5 & 0 & 6,51 \\
\hline 14400 & 1566 & 156,6 & 1252,8 & 0,00 \\
\hline 4800 & 2345 & 234,5 & 703,5 & 4,89 \\
\hline 16800 & 345 & 34,5 & 241,5 & 0,00 \\
\hline 16800 & 23 & 2,3 & 16,1 & 0,08 \\
\hline 19200 & 4567 & 456,7 & 0 & 16,91 \\
\hline 19200 & 2345 & 234,5 & 1407 & 8,55 \\
\hline 19200 & 6754 & 675,4 & 5403,2 & 14,07 \\
\hline 14400 & 9876 & 987,6 & 3950,4 & 35,27 \\
\hline 14400 & 125 & 12,5 & 25 & 0,46 \\
\hline 4800 & 3456 & 345,6 & 345,6 & 9,60 \\
\hline 21600 & 564 & 56,4 & 112,8 & 0,00 \\
\hline 4800 & 6785 & 678,5 & 5428 & 22,62 \\
\hline 12000 & 6754 & 675,4 & 0 & 18,76 \\
\hline 7200 & 3456 & 345,6 & 1036,8 & 7,20 \\
\hline 4800 & 7654 & 765,4 & 5357,8 & 0,00 \\
\hline 4800 & 3445 & 344,5 & 2756 & 12,76 \\
\hline 7200 & 1234 & 123,4 & 617 & 4,57 \\
\hline 2400 & 9876 & 987,6 & 4938 & 36,01 \\
\hline 14400 & 3456 & 345,6 & 1036,8 & 11,52 \\
\hline 21600 & 1566 & 156,6 & 313,2 & 3,26 \\
\hline 14400 & 2345 & 234,5 & 938 & 0,00 \\
\hline 14400 & 345 & 34,5 & 138 & 0,96 \\
\hline 9600 & 23 & 2,3 & 4,6 & 0,06 \\
\hline 9600 & 4567 & 456,7 & 1370,1 & 15,22 \\
\hline 2400 & 2345 & 234,5 & 938 & 7,82 \\
\hline 7200 & 6754 & 675,4 & 4727,8 & 14,07 \\
\hline 4800 & 9876 & 987,6 & 0 & 0,00 \\
\hline 4800 & 125 & 12,5 & 100 & 0,39 \\
\hline 9600 & 3456 & 345,6 & 691,2 & 9,60 \\
\hline 7200 & 564 & 56,4 & 282 & 2,09 \\
\hline 12000 & 6785 & 678,5 & 0 & 14,14 \\
\hline 12000 & 6754 & 675,4 & 5403,2 & 21,11 \\
\hline 19200 & 3456 & 345,6 & 1036,8 & 12,34 \\
\hline 16800 & 7654 & 765,4 & 2296,2 & 21,26 \\
\hline 7200 & 3445 & 344,5 & 0 & 11,96 \\
\hline 12000 & 1234 & 123,4 & 123,4 & 4,57 \\
\hline 2400 & 9876 & 987,6 & 3950,4 & 36,58 \\
\hline 21600 & 3456 & 345,6 & 1728 & 12,00 \\
\hline 4800 & 8766 & 876,6 & 5259,6 & 0,00 \\
\hline 14400 & 543 & 54,3 & 325,8 & 1,13 \\
\hline 21600 & 5667 & 566,7 & 3400,2 & 17,71 \\
\hline 16800 & 5439 & 543,9 & 3263,4 & 18,13 \\
\hline 19200 & 2345 & 234,5 & 0 & 8,55 \\
\hline 2400 & 6545 & 654,5 & 5236 & 23,86 \\
\hline 16800 & 876 & 87,6 & 613,2 & 0,00 \\
\hline 9600 & 7654 & 765,4 & 0 & 23,92 \\
\hline
\end{tabular}

Fig. 4. Experiment Data. Part 2

Acknowledgement. This research is supported by research project TIN201565515-C4-1-R from the Spanish government. 


\section{References}

1. Javier Alonso-Mora, Samitha Samaranayake, Alex Wallar, Emilio Frazzoli, and Daniela Rus. On-demand high-capacity ride-sharing via dynamic trip-vehicle assignment. Proceedings of the National Academy of Sciences, 114(3):462-467, 2017.

2. L. Bianchi, M. Dorigo, L.M. Gambardella, and W.J. Gutjahr. A survey on metaheuristics for stochastic combinatorial optimization. Natural Computing, 8(2):239$287,2009$.

3. M. Buning and W. Schonewolf. Freight transport system for urban shipment and delivery. 2011 IEEE Forum Integr. Sustain. Transp. Syst. FISTS 2011, pages 136$140,2011$.

4. Gentile, Guido and Noekel, Klaus. Modeling Public Transport Passenger Flows in the Era of Intelligent Transport Systems. Springer, 2016.

5. Gonzalez-Feliu, J. and Semet, F. and Routhier, J.L. Sustainable Urban Logistics: Concepts, Methods and Information Systems. Springer, 2014.

6. S.E. Griffis, T.J. Goldsby, M. Cooper, and D.J. Closs. Aligning logistics performance measures to the information needs of the firm. Journal of Business Logistics, $48: 35-56,2007$.

7. A. Gunasekaran and B. Kobu. Performance measures and metrics in logistics and supply chain management: a review of recent literature (1995-2004) for research and applications. Int. Journal of Production Research, 45:2819-2840, 2007.

8. C. Macharis and S. Melo. City distribution and Urban freight transport: Multiple perspectives. Edward Elgar Publishing, 2011.

9. J. Morana and J. Gonzalez-Feliu. A sustainable urban logistics dashboard from the perspective of a group of operational managers. Management Research Review, 38(10):1068-1085, 2015.

10. Paolo Neirotti, Alberto De Marco, Anna Corinna Cagliano, Giulio Mangano, and Francesco Scorrano. Current trends in smart city initiatives: Some stylised facts. Cities, 38:25 - 36, 2014.

11. M. Pagell and Z. Wu. Building a more complete theory of sustainable supply chain management using case studies of 10 exemplars. Journal of Supply Chain Management, 45:37-56, 2009.

12. Chatterjee Rajeshwari. Optimizing last mile delivery using public transport with multiagent based control. Master Thesis, pages 1-59, 2016.

13. Market Reports. Global last mile delivery market size, status and forecast 20192025. The Market Reports. Report Code : 1362721, pages 1-114, 2019.

14. J. Sabater and C. Sierra. Review on computational trust and reputation models. Artificial Intelligence Review, 24(1):33-60, 2005.

15. Ryan L Skiver and Michael Godfrey. Crowdserving: A last mile delivery method for brick-and-mortar retailers. Global Journal of Business Research, 11(2):67-77, 2017.

16. Zuopeng Xiao, James J Wang, James Lenzer, and Yonghai Sun. Understanding the diversity of final delivery solutions for online retailing: A case of shenzhen, china. Transportation Research Procedia, 25:985 - 998, 2017. World Conference on Transport Research - WCTR 2016 Shanghai. 10-15 July 2016. 